\title{
Deficiencies in locating the cricothyroid membrane by palpation: We can't and the surgeons can't, so what now for the emergency surgical airway?
}

\author{
J. Adam Law, MD
}

Received: 31 January 2016/Revised: 8 February 2016/ Accepted: 5 April 2016/Published online: 5 May 2016

(C) Canadian Anesthesiologists' Society 2016

At times, it seems that we were never meant to discover the underlying mysteries of the neck's surface anatomy. The quest first began with the cricoid cartilage and although Sellick's maneuver ${ }^{1}$ seemed an acceptable concept, it eventually proved somewhat disappointing in its application. Who knows how often this technique actually hit its target? Now, as Hiller et al. outline in this issue of the Journal, just north of the cricoid cartilage and sorely needed for expeditious emergency surgical airway (ESA), it seems that the cricothyroid membrane (CTM) is also keeping its exact location a closely guarded secret. ${ }^{2}$

The ESA is infrequently performed by the average anesthesiologist and has had its issues. Historically, in a can't intubate, can't oxygenate (CICO) emergency, practitioners been slow to initiate the procedure. Furthermore, some of the techniques used to execute the ESA have been less than effective, perhaps accounting for the generally poor success rates. ${ }^{3,4}$ Fortunately, recently published national airway guidelines address these matters with more definitive recommendations for both when and how ESA should occur. ${ }^{5-7}$ The trouble is, establishing an ESA generally begins with identifying the location of the

Work supported by the Department of Anesthesia, Dalhousie University.

Travaux soutenus par le département d'anesthésie de l'Université Dalhousie.

\section{J. A. Law, MD ( $\square)$}

Department of Anesthesia, Pain Management and Perioperative Medicine, Faculty of Medicine, QEII Health Sciences Centre, Halifax Infirmary Site, Dalhousie University, 1796 Summer

Street, Halifax, NS B3H 3A7, Canada

e-mail: jlaw@dal.ca
CTM by external palpation. As it turns out, for the life of us (not to mention that of our patients), it seems that we can't reliably accomplish this task.

At least six recently published studies from a variety of countries attest to this conclusion. ${ }^{8-13}$ These studies used similar methodologies to determine how often physician assessors correctly identified the location of the CTM by external palpation. The assessors' estimations in these studies (and similarly in the present one) were compared with the actual location of the CTM as confirmed by ultrasound. In most studies, the assessor's attempt to localize the CTM was defined as a failure if the estimation was either outside the vertical confines of the CTM or $>5$ $\mathrm{mm}$ from the midline. The results are sobering. Correctly identifying the CTM location using external palpation is no better than a 50:50 proposition, give or take, even in nonobese subjects. Most of the studies include scatter plots of the assessors' estimates, and in some cases, localization is off by at least $3 \mathrm{~cm}$ above, below, or lateral to the actual CTM location. Not surprisingly, there is a trend towards worse results in females and obese subjects given their less distinct thyroid cartilage anatomy. These findings occurred in the relatively stress-free conditions of a clinical study in volunteer subjects. We can probably expect even worse results when we add the anxiety-related performance degradation in an actual CICO situation.

By adding trauma surgeons to the mix, Hiller et al. add fuel to the fire with their study of the accuracy of CTM identification by external palpation in female subjects. ${ }^{2}$ Notwithstanding their greater experience in performing ESA, the surgeons failed to demonstrate a significantly better success rate at CTM identification than their anesthesia provider colleagues-their absolute success rate was only $26 \%$. Indeed, the only assessor in the Hiller study to identify the CTM location correctly in all 
four of the study subjects was a first-year anesthesia trainee!

Clearly, there is major cause for concern-an infrequently performed yet potentially life-saving procedure begins with a first step that, when inaccurate, could doom the subsequent steps to failure. This raises a number of questions. Should we continue to recommend cricothyrotomy in the CICO situation? If so, are studies of CTM identification asking the right questions? Finally, given the current published evidence, how should we adapt our practice?

\section{Cricothyrotomy in CICO: Are we recommending the correct procedure?}

Many current guidelines recommend cricothyrotomy over tracheotomy for ESA in the adult patient. ${ }^{5,6}$ That said, the goal of ESA in CICO is rapid restoration of patient oxygenation. As long as it succeeds, whether it's done through the CTM or between tracheal rings is probably of secondary importance. A retrospective review of 34 ESAs performed over six years in a single United States Level 1 trauma and burn centre found that only ten $(29 \%)$ were cricothyrotomies, while the remaining $24(71 \%)$ were tracheotomies. Thirty $(88 \%)$ of the 34 ESAs were performed by surgeons. There were no complications noted in either group. ${ }^{14}$ Thus, to a surgeon familiar with the anatomy and with ready access to surgical instruments, perhaps tracheotomy is acceptable for ESA, provided time to completion corresponds with that for cricothyrotomy. For the rest of us (leaving aside the alleged potential for easy CTM localization by palpation), cricothyrotomy is probably advantageous in the adult patient. This premise is based on the trachea's more superficial position in the neck at this level, together with the protection against inadvertent tracheal injury afforded by the posterior and lateral boundaries of the signet ring shape of the cricoid cartilage and the inferior horns of the thyroid cartilage. This location may be less vascular than more distal sites, but in a living patient, active bleeding must be expected regardless of the incision location. On balance, for anesthesiologists, based on anatomic considerations and published recommendations, cricothyrotomy probably still makes sense for ESA.

\section{Cricothyroid membrane identification by external palpation: Are we asking the right questions?}

There are some questions about CTM localization that remain unanswered. It's now clear that external palpation wants for accuracy regardless of the specialty of the assessing physician. This means that techniques involving direct access to the trachea through the externally palpated CTM (e.g., Seldinger- or trocar-based percutaneous techniques; scalpel-based access using a single horizontal stab) all run the risk of malpositioning. The alternative, when using these (and indeed all) cricothyrotomy techniques, is to begin with a midline $3-4 \mathrm{~cm}$ vertical incision through skin and subcutaneous tissues over the estimated location of the CTM. ${ }^{5,6}$ The CTM is then repalpated within the wound, and its location may be far easier to determine with substantially less soft tissue between the palpating digit and the CTM. Cricothyrotomy can then proceed using the chosen technique with greater assurance of correct placement. In the author's (cadaverbased) experience, the CTM is much easier to identify during this interim "dissection" step, though published clinical corroboration is lacking. Going forward, perhaps we should study whether external palpation (or visualization) will correctly estimate where the midline vertical 3-4 $\mathrm{cm}$ incision should occur, such that the CTM will be palpable within the wound in a high percentage of cases.

Secondly, given that $82 \%$ of the 185 subjects throughout the now seven studies of external CTM palpation were female (based on the assumption that the CTM location would prove more elusive in females than in males), it is unclear whether these findings are equally applicable to males. The few studies that included male subjects ${ }^{10-12}$ were not designed or powered to detect a difference in success rates between sexes. In a study by Lamb et al., ${ }^{10}$ there was a trend towards a higher success rate of CTM localization in males. Even in males of normal body habitus, this equated to only $72 \%$ and dropped to a $39 \%$ success rate in obese males. Pragmatically, even if future studies proved that CTM localization by external palpation was more accurate in males than in females, it would probably be impractical to recommend procedures for ESA that differed by sex.

Finally, and as recommended in the recently published Difficult Airway Society guidelines from the United Kingdom, ${ }^{5}$ the concept of the "laryngeal handshake" has been recommended to help identify the anatomy prior to ESA. Introduced by Dr. Richard Levitan, ${ }^{15}$ the concept espouses use of the non-dominant hand to palpate and move the hyoid bone and the thyroid and cricoid cartilage from side to side to help identify the larynx. The thyroid cartilage can then be stabilized and the CTM location sought inferiorly. While potentially helpful, this technique also awaits published clinical validation.

The verdict is in on CTM identification by external palpation, and to date, it doesn't look promising. Notwithstanding, the jury is still out on the foregoing questions, providing fertile ground for future studies. 
Given the current evidence, should existing recommendations for ESA be modified?

The study by Hiller et al. and those previously published are uniform in their message that identification of the CTM by external palpation is unreliable. This phenomenon likely persists across sexes with some variation in terms of patient body mass index and neck circumference. The consistency of the findings amongst the studies is impressive and the message cannot be ignored. There are two major implications-both involve the need for a better way to determine the CTM location.

First, prior identification of the CTM location with ultrasound is advisable ${ }^{16}$ when the management plan for an anticipated difficult airway scenario includes airway "double setup" 17 preparations for rapid ESA in case of CICO. This has implications for equipment acquisition and availability as well as for staff training. To avoid delay in performing ESA once a CICO situation is underway, the use of ultrasound cannot be espoused unless the system is already present and booted.

Second, regardless of the chosen technique or device, ESA by cricothyrotomy should probably always begin with the aforementioned midline $3-4 \mathrm{~cm}$ vertical incision through skin and subcutaneous tissues over the estimated location of the CTM. This allows for more precise determination of the CTM location within the wound. Thus, based on the now published evidence, existing guidelines that recommend a single horizontal stab incision from skin to trachea through the CTM-when the cricothyroid membrane is (ostensibly) palpable externally- ${ }^{5,18}$ would need modification. In many cases, the "slash" would likely occur in the wrong location. This would likewise apply to percutaneous Seldinger- or trocarbased techniques.

With good decision-making in the anticipated difficult airway situation and a well thought out approach to the unanticipated difficult airway, the need for establishing an ESA will continue to remain a rare event. Nevertheless, this will always be a double-edged sword-i.e., good that we may never have to do it, but bad in that we'll never have a chance to master it through experience. This means that, both mentally ${ }^{19}$ and in simulation sessions, we must continue to rehearse the management techniques of a CICO scenario, including optimizing face mask ventilation; placing a supraglottic device, if not already attempted; ensuring pharmacologic paralysis if there is no prospect of a timely return to spontaneous ventilation; and performing ESA without delay. ${ }^{6}$ Furthermore, when informed by the results of continuing investigations, we must continue to adapt to emerging evidence by modifying existing guidelines and recommendations where needed.

\section{Incapables de localiser la membrane cricothyroïdienne par palpation: si nous n'y parvenons pas et les chirurgiens non plus, que faire alors en cas de voies aériennes chirurgicales d'urgence?}

Il semble parfois que nous n'étions pas supposés découvrir les mystères sous-jacents à l'anatomie superficielle du cou. La quête a débuté avec le cartilage cricoïde et, bien que la manœuvre de Sellick ${ }^{1}$ ait semblé être un concept acceptable, elle s'est finalement révélée quelque peu décevante dans son application. Qui sait à quelle fréquence cette technique a véritablement réussi? Aujourd'hui, comme Hiller et coll. le décrivent dans ce numéro du Journal, la membrane cricothyroïdienne, située juste au nord du cartilage cricoïde et dont on a le plus grand besoin quand il s'agit d'obtenir rapidement un accès chirurgical d'urgence aux voies aériennes, semble elle aussi bien garder le secret de son emplacement exact. ${ }^{2}$

L'anesthésiologiste moyen a rarement besoin d'obtenir un accès chirurgical d'urgence aux voies aériennes, lequel s'accompagne de son lot de problèmes. Historiquement, dans une situation d'urgence où il est «impossible d'intuber, impossible d'oxygéner », la plupart des praticiens sont réticents à entreprendre cette intervention. À cela s'ajoute le fait que certaines des techniques employées pour obtenir un accès chirurgical d'urgence aux voies aériennes se sont avérées loin d'être efficaces - ce qui explique peut-être le faible taux de réussite global. ${ }^{3,4}$ Heureusement, les recommandations nationales sur les voies aériennes publiées récemment abordent ces questions et proposent des recommandations plus définitives quant au moment et à la manière d'entreprendre un accès chirurgical d'urgence aux voies aériennes. ${ }^{5-7}$ Le problème, c'est que pour établir un accès chirurgical d'urgence aux voies aériennes, on commence en général par localiser la membrane cricothyroïdienne par palpation externe. Mais apparemment, malgré tous nos efforts (et sans mentionner notre inquiétude pour la survie de nos patients), il semble que nous ne soyons pas capables d'accomplir ce geste de façon fiable.

Au moins six études publiées récemment dans divers pays en attestent. ${ }^{8-13}$ Ces études se fondent sur des méthodologies semblables afin de déterminer la fréquence à laquelle les médecins évaluateurs ont correctement localisé la membrane cricothyroïdienne par palpation externe. Les estimations des évaluateurs dans ces études (tout comme dans l'étude publiée ici) ont été comparées à 
l'emplacement réel de la membrane cricothyroïdienne déterminé par échoguidage. Dans la plupart des études, la tentative de l'évaluateur pour localiser la membrane cricothyroïdienne a été définie comme un échec si l'estimation se situait hors des limites verticales de la membrane cricothyrö̈dienne ou à $>5 \mathrm{~mm}$ de la ligne médiane. Les résultats sont consternants. L'identification correcte de l'emplacement de la membrane cricothyroïdienne par palpation externe se fait dans plus ou moins $50 \%$ des cas et ce, même chez les patients non obèses. La plupart des études présentent un diagramme de dispersion des estimations des évaluateurs et, dans certains cas, la localisation est erronée d'au moins $3 \mathrm{~cm}$ au-dessus, au-dessous ou latéralement à la position réelle de la membrane cricothyroïdienne. Constat peu surprenant, les résultats les pires ont tendance à s'observer chez les patients de sexe féminin ou obèses, étant donné leur anatomie moins distinctive du cartilage thyroïdien. Ces résultats ont été enregistrés dans des conditions relativement peu stressantes, c'est-à-dire dans le cadre d'une étude clinique menée auprès de volontaires. Nous pouvons probablement nous attendre à des résultats encore plus affligeants si l'on ajoute la détérioration de la performance liée à l'anxiété dans une situation réelle où il est «impossible d'intuber, impossible de ventiler ».

En ajoutant les chirurgiens de traumatisme à leur étude, Hiller et coll. mettent de l'huile sur le feu de leur évaluation de la précision de localisation de la membrane cricothyroïdienne par palpation externe chez les patientes femmes. ${ }^{2}$ Malgré leur supplément d'expérience en matière d'ouverture d'accès chirurgical d'urgence aux voies aériennes, les chirurgiens n'ont cependant pas réussi à démontrer un taux de réussite significativement plus élevé pour localiser la membrane cricothyroïdienne que leurs collègues anesthésiologistes - leur taux de réussite absolu n'était que de $26 \%$. En fait, dans l'étude de Hiller, le seul évaluateur à localiser correctement la membrane cricothyrö̈dienne chez les quatre patients à l'étude était un stagiaire en anesthésie de première année!

Il ne fait aucun doute que cet état de fait est très inquiétant - une intervention peu fréquente mais qui peut potentiellement sauver des vies avec une première étape qui, si inexacte, peut vouer à l'échec toutes les étapes subséquentes. Cela soulève un certain nombre de questions. Devrions-nous continuer de recommander la cricothyrotomie lors de situations «impossible d'intuber, impossible d'oxygéner »? Le cas échéant, les études sur la localisation de la membrane cricothyroïdienne posent-elles véritablement les bonnes questions? Enfin, au vu des données probantes actuellement publiées, comment devrions-nous adapter notre pratique?
La cricothyrotomie dans les situations « impossible d'intuber, impossible d'oxygéner »: recommandons-nous les bons gestes?

Plusieurs directives actuelles recommandent la cricothyrotomie plutôt que la trachéotomie pour obtenir un accès chirurgical d'urgence aux voies aériennes chez le patient adulte. ${ }^{5,6}$ Ceci étant dit, le rétablissement rapide de l'oxygénation du patient est l'objectif premier d'un accès chirurgical d'urgence aux voies aériennes en situation «impossible d'intuber, impossible d'oxygéner». Tant qu'on y parvient, la voie d'accès importe probablement peu - que ce soit via la membrane cricothyroïdienne ou entre les anneaux trachéaux. Un compte rendu rétrospectif de 34 accès chirurgicaux d'urgence aux voies aériennes réalisés au cours d'une période de six ans dans un seul centre de traumatologie et grands brûlés de niveau 1 aux États-Unis a observé que seuls dix $(29 \%)$ de ces accès ont été obtenus par cricothyrotomie, alors que les 24 autres (71\%) étaient des trachéotomies. Trente ( $88 \%$ ) des 34 accès chirurgicaux d'urgence aux voies aériennes ont été réalisés par des chirurgiens. Aucune complication n'a été enregistrée dans les deux groupes. ${ }^{14}$ Ainsi, si un chirurgien connait bien l'anatomie et dispose d'instruments chirurgicaux à portée de main, peut-être que la trachéotomie est acceptable pour obtenir un accès chirurgical d'urgence aux voies aériennes - tant que la durée nécessaire à sa réalisation est égale à celle nécessaire à réaliser une cricothyrotomie. Pour tous les autres intervenants (en laissant de côté le potentiel allégué de localisation facile de la membrane cricothyroïdienne par palpation), la cricothyrotomie constitue probablement le meilleur choix chez un patient adulte. Cette prémisse s'appuie sur la position plus superficielle de la trachée à ce niveau du cou, en plus de la protection contre une lésion accidentelle à la trachée qu'offrent les limites postérieures et latérales de la forme en anneau du cartilage cricoïde et les cornes inférieures du cartilage thyroïde. De plus, cet emplacement est probablement moins vascularisé que d'autres sites plus distaux, bien qu'il faille s'attendre à des saignements indépendamment du site d'incision chez un patient vivant. C'est pourquoi, si l'on se fonde sur l'anatomie et les recommandations publiées, la cricothyrotomie reste probablement le meilleur choix pour un accès chirurgical d'urgence aux voies aériennes pour un anesthésiologiste, toutes choses considérées.

\section{L'identification de la membrane cricothyroïdienne par palpation externe: pose-t-on les bonnes questions?}

Certaines questions concernant la localisation de la membrane cricothyroïdienne demeurent sans réponse. 
Nous savons désormais que la palpation externe manque de précision, indépendamment de la spécialité du médecin évaluateur. En d'autres termes, les techniques qui s'appuient sur un accès direct à la trachée via la membrane cricothyroïdienne palpée depuis l'extérieur (par ex., les techniques percutanées de Seldinger ou réalisées à l'aide d'un trocart, un accès basé sur un scalpel à l'aide d'une coupure horizontale unique) courent toutes le risque d'un mauvais positionnement. L'alternative, lorsqu' on a recours à ces techniques de cricothyrotomie (en fait, à toutes ces techniques), est de commencer par une incision verticale médiane de 3-4 cm à travers la peau et les tissus sous-cutanés situés sur l'emplacement estimé de la membrane cricothyrö̈dienne. ${ }^{5,6}$ La membrane cricothyrö̈dienne est ensuite palpée à nouveau dans la lésion, et son emplacement devrait être bien plus facile à déterminer lorsqu'il y a considérablement moins de tissu mou entre le doigt qui palpe et la membrane cricothyrö̈dienne. La cricothyrotomie peut ensuite se faire à l'aide de la technique choisie et en étant absolument certain de son bon positionnement. Selon mon expérience (acquise sur des cadavres), la membrane cricothyroïdienne est bien plus facile à identifier pendant cette étape intérimaire de « dissection », bien qu'il n'y ait pas de données probantes cliniques publiées corroborant cette observation. À l'avenir, peut-être devrions-nous examiner si la palpation externe (ou la visualisation) permet d'estimer correctement le lieu de l'incision verticale médiane de $3-4 \mathrm{~cm}$ de façon à ce que la membrane cricothyroïdienne soit palpable dans la lésion dans un pourcentage élevé des cas.

Deuxièmement, étant donné que $82 \%$ des 185 patients dans les sept études de palpation externe de la membrane cricothyroïdienne étaient des femmes (selon l'hypothèse que la localisation de la membrane cricothyrö̈dienne est plus difficile chez la femme que chez l'homme), nous ne savons pas si nous pouvons appliquer ces observations à des patients mâles. Les quelques études ayant inclus des patients de sexe masculin $^{10-12}$ n'ont pas été conçues ni n'avaient pas suffisamment de puissance pour détecter une quelconque différence entre les sexes en matière de taux de réussite. Dans une étude de Lamb et coll., ${ }^{10}$ les auteurs ont observé une tendance vers un taux de réussite plus élevé de localisation de la membrane cricothyrö̈dienne chez les hommes. Cependant, même chez des hommes de corpulence normale, cela équivalait à seulement $72 \%$ et ce taux a chuté à $39 \%$ en cas d'obésité. De façon pragmatique, même si les études futures parvenaient à prouver que la localisation de la membrane cricothyroïdienne par palpation externe était plus précise chez les hommes que chez les femmes, il serait probablement peu pratique de recommander des interventions différentes d'accès chirurgical d'urgence aux voies aériennes selon le sexe du patient.
Enfin, et comme le recommandent les directives de la Société pour des voies aériennes difficiles (Difficult Airway Society) récemment publiées au Royaume-Uni, ${ }^{5}$ le concept d'une «poignée de main laryngée » a été recommandé pour aider à identifier l'anatomie avant d'obtenir un accès chirurgical d'urgence aux voies aériennes. Le concept a été introduit par le Dr Richard Levitan, et préconise l'usage de la main non dominante pour palper et mobiliser l'os hyoïde, la thyroïde et le cartilage cricoïde de part et d'autre afin d'identifier le larynx. Le cartilage thyroïde peut ensuite être stabilisé et on peut chercher à localiser la membrane cricothyrö̈dienne au-dessous. Bien qu'elle puisse être utile, cette technique n'a pas encore été validée par des études cliniques publiées.

Le verdict est rendu concernant l'identification de la membrane cricothyroïdienne par palpation externe et, à date, il n'est guère prometteur. Ceci étant dit, le jury délibère encore concernant les questions soulevées ci-dessus, lesquelles constituent indubitablement un terreau fertile pour des études futures.

\section{Étant donné les données probantes actuelles, faut-il modifier les recommandations existantes concernant l'accès chirurgical d'urgence aux voies aériennes?}

L'étude de Hiller et coll. et ses prédécesseures sont unanimes : l'identification de la membrane cricothyrö̈dienne par palpation externe n'est pas fiable. Ce phénomène existe probablement tant chez les patients de sexe masculin que féminin et varie en termes d'indice de masse corporel et de circonférence du cou du patient. La cohérence des résultats d'une étude à l'autre est impressionnante et le message ne peut être ignoré. Il y a deux implications majeures, et toutes deux soulignent le besoin d'une meilleure façon de déterminer l'emplacement de la membrane cricothyrö̈dienne.

En premier lieu, l'identification préalable de l'emplacement de la membrane cricothyroïdienne par échoguidage est recommandée ${ }^{16}$ lorsque le plan de prise en charge d'un cas anticipé de voies aériennes difficiles inclut des préparations de «double installation » ${ }^{17}$ des voies aériennes pour obtenir un accès chirurgical d'urgence aux voies aériennes dans une situation «impossible d'intuber, impossible d'oxygéner ». Ceci a un impact sur l'acquisition du matériel et sa disponibilité ainsi que sur la formation du personnel. Afin d'éviter tout retard dans l'obtention d'un accès chirurgical d'urgence aux voies aériennes une fois qu'une situation «impossible d'intuber, impossible d'oxygéner » est identifiée, le recours à l'échoguidage ne devrait être recommandé que si le système est déjà fonctionnel en salle d'opération.

Deuxièmement, indépendamment de la technique ou du dispositif de choix, un accès chirurgical d'urgence aux voies 
aériennes par cricothyrotomie devrait toujours commencer par une incision verticale médiane de $3-4 \mathrm{~cm}$, tel que mentionné plus haut, à travers la peau et les tissus sous-cutanés, au niveau estimé de la membrane cricothyroïdienne. Cette étape permet de déterminer plus précisément l'emplacement de la membrane cricothyrö̈dienne dans la lésion. Ainsi, sur la base des données probantes publiées aujourd'hui, les directives existantes qui recommandent une incision horizontale unique de la peau à la trachée via la membrane cricothyroïdienne - lorsque la membrane cricothyroïdienne est (ostensiblement) palpable à l'extérieur ${ }^{5,18}$ - devraient être amendées. Dans bon nombre de cas, la «coupure» serait probablement faite au mauvais endroit. De la même façon, cela s'appliquerait aux techniques percutanées de Seldinger ainsi qu'à celles utilisant un trocart.

Le besoin d'établir un accès chirurgical d'urgence aux voies aériennes continuera d'être un cas isolé si l'on prend de bonnes décisions en situation de voies aériennes anticipées comme difficiles et si l'on dispose d'une approche bien pensée en cas de voies aériennes difficiles non anticipées. Toutefois, cette intervention sera toujours une épée à double tranchant : le bon côté est qu'il se peut que nous n'ayons jamais à la pratiquer, mais le mauvais côté est que nous n'aurons jamais l'occasion de la maîtriser en acquérant de l'expérience. Cela veut dire que, tant du point de vue mental ${ }^{19}$ que dans les séances de simulation, nous devons continuer à pratiquer les techniques de prise en charge d'un cas où il est «impossible d'intuber, impossible d'oxygéner », y compris l'optimisation de la ventilation au masque; le positionnement d'un dispositif supraglottique, si cela n'a pas encore été essayé; le fait de s'assurer de la paralysie pharmacologique si l'on n'anticipe pas de retour opportun à la ventilation spontanée, et l'établissement sans retard d'un accès chirurgical d'urgence aux voies aériennes. ${ }^{6}$ En outre, lorsque nous prendrons connaissance des résultats des études en cours, nous devrons continuer à nous adapter aux nouvelles données probantes en modifiant les directives et les recommandations existantes au besoin.

Conflicts of interest None declared.

Editorial responsibility This submission was handled by Dr. Hilary P. Grocott, Editor-in-Chief, Canadian Journal of Anesthesia.

\section{Conflit d'intérêt Aucun}

Responsabilité éditoriale Cet article a été traité par Dr. Hilary P. Grocott, Rédacteur en chef, Journal canadien d'anesthésie.

\section{References}

1. Sellick BA. Cricoid pressure to control regurgitation of stomach contents during induction of anaesthesia. Lancet 1961; 2: 404-6.
2. Hiller KN, Karni RJ, Cai C, Holcombe JB, Hagberg CA. Comparing anesthesia provider and trauma surgeon success rates of using palpation for cricothyroid membrane identification in female subjects: a prospective observational study. Can J Anesth 2016; 63: this issue. DOI: 10.1007/s12630-016-0647-5.

3. Peterson GN, Domino KB, Caplan RA, Posner KL, Lee LA, Cheney $F W$. Management of the difficult airway: a closed claims analysis. Anesthesiology 2005; 103: 33-9.

4. Frerk C, Cook T. Management of the can't intubate can't ventilate' situation and the emergency surgical airway. In: Cook T, Woodall NM, Frerk C, editors. 4th National Audit Project of the Royal College of Anaesthetists and the Difficult Airway Society - Major Complications of Airway Management in the United Kingdom. Report and findings. London: Royal College of Anaesthetists; 2011 .

5. Frerk C, Mitchell VS, McNarry AF, et al. Difficult Airway Society 2015 guidelines for management of unanticipated difficult intubation in adults. Br J Anaesth 2015; 115: 827-48.

6. Law JA, Broemling N, Cooper RM, et al. The difficult airway with recommendations for management-part 1-difficult tracheal intubation encountered in an unconscious/induced patient. Can J Anesth 2013; 60: 1089-118.

7. Apfelbaum JL, Hagberg CA, Caplan RA, et al. Practice guidelines for management of the difficult airway: an updated report by the American Society of Anesthesiologists Task Force on Management of the Difficult Airway. Anesthesiology 2013; 118: 251-70.

8. Aslani A, Ng SC, Hurley M, McCarthy KF, McNicholas M, McCaul CL. Accuracy of identification of the cricothyroid membrane in female subjects using palpation: an observational study. Anesth Analg 2012; 114: 987-92.

9. You-Ten KE, Desai D, Postonogova T, Siddiqui N. Accuracy of conventional digital palpation and ultrasound of the cricothyroid membrane in obese women in labour. Anaesthesia 2015; 70: 1230-4.

10. Lamb A, Zhang J, Hung $O$, et al. Accuracy of identifying the cricothyroid membrane by anesthesia trainees and staff in a Canadian institution. Can J Anesth 2015; 62: 495-503.

11. Elliott DS, Baker PA, Scott MR, Birch CW, Thompson JM. Accuracy of surface landmark identification for cannula cricothyroidotomy. Anaesthesia 2010; 65: 889-94.

12. Bair AE, Chima $R$. The inaccuracy of using landmark techniques for cricothyroid membrane identification: a comparison of three techniques. Acad Emerg Med 2015; 22: 908-14.

13. Kristensen MS, Teoh WH, Rudolph SS, et al. Structured approach to ultrasound-guided identification of the cricothyroid membrane: a randomized comparison with the palpation method in the morbidly obese. Br J Anaesth 2015; 114: 1003-4.

14. Dillon JK, Christensen B, Fairbanks T, Jurkovich G, Moe KS. The emergent surgical airway: cricothyrotomy vs. tracheotomy. Int J Oral Maxillofac Surg 2013; 42: 204-8.

15. Levitan $R$. Available from: URL: http://www.airwaycam/ cricothyrotomy/ (accessed February 2016).

16. Kristensen $M S$. Ultrasonography in the management of the airway. Acta Anaesthesiol Scand 2011; 55: 1155-73.

17. Law JA, Broemling N, Cooper RM, et al. The difficult airway with recommendations for management-part 2-the anticipated difficult airway. Can J Anesth 2013; 60: 1119-38.

18. Kristensen MS, Teoh WH, Baker PA. Percutaneous emergency airway access; prevention, preparation, technique and training. $\mathrm{Br}$ J Anaesth 2015; 114: 357-61.

19. Brindley $P G$, Beed $M$, Duggan LV, Hung O, Murphy MF. Updating our approach to the difficult and failed airways: time to "stop and think". Can J Anesth 2016; DOI: 10.1007/s12630-0160594-1. 